\title{
Corela
}

Cognition, représentation, langage

HS-30 | 2020

Phonétique, littérature et enseignement du FLE :

théories et recherches

\section{Identifier les contenus phonétiques d'un texte littéraire}

Bertrand Lauret

\section{(2) OpenEdition}

Journals

Édition électronique

URL : https://journals.openedition.org/corela/10001

DOI : 10.4000/corela.10001

ISSN : 1638-573X

Éditeur

Cercle linguistique du Centre et de l'Ouest - CerLICO

Référence électronique

Bertrand Lauret, «Identifier les contenus phonétiques d'un texte littéraire », Corela [En ligne], HS-30 |

2020, mis en ligne le 24 juin 2020, consulté le 18 juin 2021. URL : http://journals.openedition.org/ corela/10001; DOI : https://doi.org/10.4000/corela.10001

Ce document a été généré automatiquement le 18 juin 2021.

\section{(c) $)(1)(2)$}

Corela - cognition, représentation, langage est mis à disposition selon les termes de la licence Creative Commons Attribution - Pas d'Utilisation Commerciale - Partage dans les Mêmes Conditions 4.0 International. 


\title{
Identifier les contenus phonétiques d'un texte littéraire
}

\author{
Bertrand Lauret
}

Instruction officielle du 13 septembre 1890 :

« L'étude de la langue doit précéder l'étude

littéraire " (titre du chapitre 1$)$ :

«Vouloir unir prématurément l'étude littéraire à

l'étude de la langue, c'est tout compromettre à la

fois, c'est s'exposer à ne jamais lire couramment

la langue, à ne jamais la parler surtout, et à ne jamais goûter la littérature dans ce qu'elle a de réellement original, c'est-à-dire dans ce qui en fait le véritable intérêt. S'il fallait sacrifier l'une des deux études à l'autre, il serait encore préférable de s'en tenir modestement à la langue et de réserver la littérature pour un âge où l'esprit a conquis, avec sa maturité, sa liberté d'allure, sa souplesse et son indépendance ».

(Puren, $1988: 77$ )

\section{Introduction}

1 Parmi les supports à l'entraînement phonétique, le texte littéraire semble aujourd'hui des moins adéquats. Les développements méthodologiques en didactique de la phonétique ont traité la perception, avec la méthode verbo-tonale (Renard, 1979), la production avec la méthode articulatoire (Bernstein, 1975), l'oralité pour fédérer perception et production dans la notion de "paysage sonore» (Lhote, 2001) pour ne citer que trois approches. Ces développements se sont intéressés aux éléments suprasegmentaux - en particulier le découpage en groupes rythmiques ou mots phonétiques, mais aussi la syllabation (Pagel et al., 2012) -,et aux unités segmentales. Ces travaux concernent tout enseignant de langue dès le début de l'apprentissage car 
ils peuvent donner lieu à des choix radicaux : l'oral intégral en fait partie (Delattre, 1944). Mais, la connaissance de ces travaux didactiques favorise aussi une conscience aigue de la place respective de l'oral et de l'écrit (Weber, 2013), tant en compréhension qu'en production.

2 Le texte littéraire est aussi un corpus linguistique. Un corpus généralement écrit, mais qui peut être oralisé. Comment identifier les contenus phonétiques d'un texte littéraire au-delà des " sonorités de la phonétique dans un poème » ? C'est à une réflexion autour de l'exploitation phonétique du texte littéraire et sur des outils pratiques d'analyse qu'invite le présent article.

\section{Oral vs écrit : ambiguïté linguistique}

3 La phonétique, domaine de la linguistique consacré à la forme orale de la langue a toujours entretenu des rapports ambigus avec l'écrit. Quelques exemples :

on établit traditionnellement les premiers textes de descriptions phonétiques à l'époque du grammairien de l'Inde antique Panini (560-480 av. J.-C.), pour permettre la récitation précise des textes sacrés écrits. Force est de constater que des textes issus de traditions orales se sont servis dès que possible de l'écrit pour fixer une prononciation de référence ;

une des premières mesures de l'Association de Phonétique Internationale en 1886, association issue d'enseignants d'anglais et de français (deux langues dont les orthographes sont peu phonétiques), a été de créer... un alphabet ;

encore aujourd'hui, la transcription phonétique consiste le plus souvent en une transcription du niveau segmental, voyelles et consonnes, ce qui est à l'origine la vocation de l'orthographe. L'utilisation des symboles diacritiques suprasegmentaux ou prosodiques est beaucoup moins commune, mises à part l'indication des frontières de groupes rythmiques (ou mots phonétiques) et la syllabe accentuée en anglais ;

il aura fallu attendre les années 1980 pour voir se développer une linguistique de l'oral (Gadet, 1989 ; Morel, 1998), dont l'alphabet phonétique n'était pas toujours l'outil principal. En phonétique expérimentale apparaît alors la notion de «parole spontanée » ou oral authentique, opposée à la "parole de laboratoire », oralisation de phrases écrites.

4 On le voit donc, il semble difficile, dès que l'on dispose de l'écrit, de s'en dégager pour aborder l'oral. C'est ce que me rapportent les enseignants de langue : «Dès le début de leur apprentissage, les étudiants veulent savoir comment ça s'écrit ».

5 À l'inverse, on pourrait évoquer les rapports que le texte littéraire entretient avec l'oralité, qu'il s'agisse du discours indirect, des dialogues, des aménagements de l'orthographe pour transcrire les marques d'oralité ou même les accents d'une façon pas toujours subtile ( $«-$ Che fitrais edre assez ruche bir te vaire fifre tu les churs gomme ça... répondit mélancoliquement le bon Allemand.» (Balzac, 1848); l'accent d'un officier prussien: "-Ché bris ces bolissons de Français bar les oreilles" (Maupassant, 1883). Jusqu'à des caractéristiques propres à l'oralisation d'un certain type de textes (par exemple le « e » caduc prononcé en chanson - « Frère Jacques » - et en vers, la diérèse en vers).

6 Rappelons pour conclure ce rapide survol que l'oral préexiste à l'écrit et qu'avant qu'une communauté compte plus de lecteurs que d'auditeurs, l'écrit est destiné à être oralisé. Seules les communautés de lecteurs lisent en silence. 


\title{
3. Oral vs écrit : ambiguïté pédagogique
}

\author{
« Malgré la présence d'exercices de \\ prononciation au début de l'apprentissage mais \\ qui sont en réalité tous orientés vers l'oralisation \\ de l'écrit comme la récitation de textes écrits, \\ puis la lecture à haute voix des textes d'auteurs, \\ c'est l'enseignement de l'écrit qui prédomine et \\ qui s'effectue selon la gradation mot-phrase- \\ texte ${ }^{1}$.» (Cuq, $\left.2002: 235\right)$.
}

7 Dans l'enseignement général de la langue, les rapports conflictuels entre oral et écrit demeurent. Tant dans les institutions d'enseignement qui ont, par tradition, toujours valorisé l'écrit, en particulier pour l'accès aux textes littéraires qui était un des objectifs annoncés de l'apprentissage des langues. Le texte littéraire est alors considéré comme le meilleur de la langue, avec un lexique et une syntaxe élaborée, là où la langue orale quotidienne est jugée moins noble. Rares sont encore les institutions qui accordent une vraie place à la production orale et à la prononciation, aussi bien dans les formations que dans l'évaluation'2.

8 Les rapports conflictuels entre oral et écrit se retrouvent aussi dans les manuels, issus d'éditeurs de livres écrits, privilégiant encore le papier et l'écrit face à l'audio et à la vidéo, même si les nouvelles technologies facilitent considérablement le multimédia.

Oral et écrit s'affrontent enfin aussi dans l'espace de la classe pour les enseignants, qui doivent faire le choix décisif des places relatives accordées à ces deux aspects de la langue. Par exemple, une séquence pédagogique commence traditionnellement par un dialogue à voir/à écouter, à comprendre, éventuellement à répéter. À quel moment l'enseignant permettra-t-il l'accès à la transcription orthographique du dialogue ? Écrira-t-il d'emblée certains mots au tableau ? Une fois l'écrit disponible, les activités et les exercices propres à l'oral ne seront-ils pas irrémédiablement influencés par le texte écrit?

10 La question du recours au support écrit orthographique est encore plus saillante dans les séquences de classe consacrées à la prononciation. On sait en effet que l'exposition à l'écrit orthographique réactive presqu'irrépressiblement les habitudes de lecture (décodage) de la langue maternelle, créant des difficultés qui viennent s'ajouter aux habitudes de prononciation (prosodie et articulation). Ainsi de nombreux praticiens ont tendance à commencer la classe par des activités d'échauffement physique et vocal et de prononciation avant d'ouvrir les manuels. Ils cherchent à différer le plus possible le recours aux supports orthographiques, certains utilisant l'Alphabet Phonétique International (A.P.I.), d'autres proposant des activités et exercices purement oraux ou avec des supports visuels. Les séquences des manuels de phonétique, malgré leur support papier, donc écrit, commencent le plus souvent par des activités purement orales: écoute, perception (discrimination, identification), production (imitations, répétitions, substitutions, transformations morpho-phonologiques, etc.) en tentant de fixer, autant que faire se peut, l'image acoustique et l'articulation des nouveaux sons, avant d'aborder leurs graphies. L'écrit, abordé dans un second temps, permet alors de nouveaux exercices de type textes à trous, dictées, lectures...

11 Si l'on aborde la question du côté de la littérature, on traite en classe de l'aspect, de la forme du texte, de l'accès au sens, d'aspects linguistiques aussi variés que 
la fonction de telle anaphore, l'antécédent de telle relative, le poids de tel adverbe, l'utilisation de tel temps verbal, etc. Mais il [l'enseignant] peut également prendre le parti d'orienter la lecture des apprenants, en demandant par exemple à ces derniers d'être attentifs à l'emploi de certaines formes conjuguées dans un conte, aux sonorités de la phonétique dans un poème, à un champ ou un registre lexical dans une prose. Les possibles, rien que pour l'approche linguistique, sont légion.

(Defays et al., 2014 : 53)

Dans les faits, parmi les aspects linguistiques, les questions phonétiques sont souvent traitées à part, comme l'illustrent deux parutions de la même maison d'édition, consacrées aux textes littéraires en classe de FLE: Le français par les textes littéraires (Bouchery, 2009) qui traite de compréhension et expression écrites sans aborder aucune question phonétique; La phonétique par les textes (Filliolet, 2010), corpus de textes uniquement poétiques " choisis en fonction des sons qu'ils contiennent", et qui passe en revue voyelles et consonnes, avec en introduction une rapide mention des phénomènes d'enchainement et de liaison. Mais ici encore, même si le support commun est le texte littéraire, les aspects phonétiques ne participent pas à l'accès au sens. Nous verrons plus loin qu'ils sont pourtant essentiels.

\section{Le texte littéraire comme document authentique}

Le document authentique est un document (visuel, audio, audiovisuel, textuel) qui n'est pas conçu pour l'exploitation pédagogique mais dont la nature et les contenus sont identifiés par un enseignant (ou un auteur de manuel) comme particulièrement intéressants dans le cadre de la classe de langue (Robert, 2002). Un document authentique conjugue un double intérêt : son adéquation à l'objectif communicatif et/ ou linguistique visé par l'enseignant; son caractère authentique est le fait d'une utilisation réelle de la langue, par opposition au document fabriqué à des fins pédagogiques.

14 Le document authentique répond avant tout à un objectif communicatif et/ou linguistique adapté à un niveau (l'utilisation du futur dans les prévisions météorologiques, de l'impératif dans les recettes de cuisine, etc.).

15 L'exploitation du document authentique, en particulier au niveau élémentaire (A1, A2), varie quelque peu d'un document didactisé ou pédagogique. Tout est utile à l'identification du support (sa forme, sa composition, etc.). On privilégie ici la découverte, le questionnement, la réflexion, la discussion. On accepte de ne pas tout expliquer, ni de tout comprendre.

Ce qui distingue le texte littéraire de la plupart des autres documents authentiques, c'est son caractère « impérissable » :

S'y expriment les aspirations pérennes des humains, échos se répondant de siècle en siècle, l'expérience subjective du monde, le rapport singulier au langage, aux savoirs constitués, aux codes sociaux, aux représentations des autres et de soimême. C'est l'un des moyens d'accès à la compréhension de formes culturelles différentes. (Blondeau et al., $2004: 3$ )

Nous retenons ici la notion de texte littéraire proposée par les auteures : «aucun texte littéraire n'est réductible à un tract politique, une recette de cuisine ou une publicité » (ibid.: 4). On pourrait ajouter les modes d'emploi, les articles de presse, les messages personnels, etc. 

l'éloigne un peu plus, et pourtant à tort, des préoccupations phonétiques.

\section{Le texte littéraire en classe de FLE}

19 Parmi les supports utilisés en classe de langue, le texte littéraire a un statut très particulier: nous l'avons vu, il est issu d'une tradition (des Belles Lettres du Moyen Âge) qui le place au sommet de la maitrise de la langue ${ }^{3}$, pour le lexique, la morphosyntaxe, l'expression, l'explication, la justification; mais, il permet également une approche de la culture, puisque le texte littéraire (le morceau choisi) illustre une vision du monde, une période, un style, etc., susceptible d'être discuté et visant à la construction d'une perception esthétique.

Fiévet $(2013: 51)$ propose deux grands axes d'exploitation du texte littéraire : un axe linguistique (repérage des faits de langue - temps, mode, etc.; compréhension de l'écrit) ; un axe littéraire (éléments préparatoires au commentaire littéraire). Sous l'axe linguistique, le découpage syntaxico-prosodique ne trouve jamais sa place. On ne sollicite la phonétique que face à la récurrence d'une rime dans un poème.

\section{Typologies des textes littéraires}

21 Nous n'aborderons pas ici l'inépuisable question des " grands genres ${ }^{4}$ » littéraires, mais nous en rappelons les formes canoniques: le conte, la nouvelle, le roman, l'essai, le discours / la leçon, la poésie, le théâtre, la bande dessinée.

Certains de ces genres textuels, même dans des registres très formels, sont conçus pour être mis en voix : le théâtre, le discours / la leçon, le conte, la poésie. L'oralisation de ces trois premiers genres va chercher à optimiser l'intelligibilité / la compréhensibilité du texte. Le théâtre opposera les monologues, les dialogues, les polylogues en valorisant l'expressivité de la communication. L'oralisation de la poésie a de plus pour mission de respecter la métrique, la rime et les autres procédés propres à la poésie. Parmi ces quatre genres, le théâtre et la poésie sont ceux les plus couramment exploités en classe de langue.

Le roman, la nouvelle, l'essai et surtout la bande dessinée sont généralement destinés à être abordés sous leur forme écrite, ils sont conçus pour l'écrit. Ils peuvent néanmoins être lus à haute voix en intégralité ${ }^{5}$ ou en extraits. Les bibliothèques sonores ont connu un important développement ces vingt dernières années, aidées par les avancées technologiques. On lit en effet à ceux qui ne savent pas ou ne peuvent pas/plus lire : les enfants, les mal voyants. Mais la plupart des lecteurs ont perdu l'habitude de la lecture à voix haute, plus encore en tant qu'interprètes qu'en tant qu'auditeurs.

L'ensemble de ces genres sollicitent diversement l'attention de l'auditeur, et engagent diversement le lecteur dans son rapport à l'oralité. Certains déclareront avoir du mal à lire du théâtre, d'autres ne prendront aucun plaisir à lire à voix haute une page de roman.

Dans tous les cas, ce qui va permettre d'accéder au sens du texte, c'est son découpage en unité de sens. 


\title{
7. Lecture silencieuse vs lecture à voix haute
}

On peut opposer deux abords d'un texte littéraire. Par la lecture du texte imprimé : « La première lecture doit être silencieuse : l'étudiant est seul face aux bruissements du texte » (Blondeau et al., 2004 : 6). Par l'écoute du texte lu : le texte est déjà oralisé par un acteur (livres audio) ou par l'enseignant qui l'a préparé. La lecture doit en faciliter l'accès : lente, groupes rythmiques marqués, mots importants mis en valeur... Il s'agit déjà d'une interprétation qui peut aider à accéder à un sens du texte.

En apprenant à lire, nous apprenons la lecture silencieuse, trois fois plus rapide que la lecture à voix haute. On lit silencieusement en moyenne 27000 mots / heure, alors qu'à haute voix, nous n'en lisons en moyenne que 9000 (Chevalier, 1992).

Le contact avec le texte littéraire se fait donc très généralement par l'écrit. On attend de l'étudiant non pas qu'il se lance de prime abord dans la compréhension du texte, mais qu'il tente de tirer un maximum d'informations sur le texte à partir de la forme, de la structure générale, du type de phrases, des modes et temps verbaux, de formes ou de mots récurrents, etc. Cette recherche globale est guidée par des questions de l'enseignant qui doivent l'aider à identifier rapidement le type de texte et des éléments essentiels de contenu. Cette recherche est généralement collective, favorisant l'échange, les questionnements, les hypothèses qui sont tout aussi importants que les réponses. Lors de ces échanges, des parties du texte sont éventuellement oralisées, généralement des syntagmes - comme des unités de sens, réponses à des questions plus que des phrases entières.

On peut se demander de quel type d'exercice relève la lecture continue à voix haute en langue étrangère. L'oralisation d'un texte écrit est-elle une activité phonétique? Elle l'est à en croire les étudiants qui placent la lecture à haute voix parmi les premières procédures classiques d'entraînement à la prononciation, au même titre que la répétition. Pour Lah (2014), la lecture favorise la formation de messages corrects et bien prononcés. Pourtant, la lecture à voix haute est une activité complexe qui exige d'une part la structuration prosodique d'un texte dont le lecteur n'est pas l'auteur, cette structuration nécessitant la plus grande anticipation possible. D'autre part, la lecture à haute voix associe les difficultés du décodage orthographique (les correspondances graphie-phonie) aux difficultés de prononciation (réalisation articulatoire). Cette complexité doit se réaliser dans l'immédiateté et la successivité.

La prosodie peut néanmoins aider le lecteur silencieux à accéder au sens du texte, tant en compréhension qu'en production.

\section{La ponctuation et le découpage syntaxico- prosodique}

\author{
« La ponctuation est l'ensemble des signes \\ conventionnels servant à indiquer, dans l'écrit, \\ des faits de langue orale comme les pauses et \\ l'intonation, ou marquer certaines coupures et \\ certains liens logiques. » (Grevisse, \\ $1993: 144 \S 115)$.
}



responsabilité des auteurs à celle des typographes. Certains opposent un usage moderne de la ponctuation qui découpe le texte en fonction de la syntaxe, alors que l'usage $d u \mathrm{xVIII}^{\mathrm{e}}$ siècle se situait "dans la perspective d'une lecture à haute voix » (Lorenceau, 1978). Nicolas Beauzée qui rédige l'article « Ponctuation » de L'Encyclopédie, précise que le choix de la ponctuation « dépend de la proportion qu'il convient d'établir dans les pauses; et cette proportion dépend de la combinaison de trois principes fondamentaux: 1) le besoin de respirer; 2) la distinction des sens partiels qui constituent le discours ; 3) la différence de degrés de subordination qui conviennent à chacun de ces sens partiels dans l'ensemble du discours " (Grammaire générale, livre III, p. 577-578, cité par Lorenceau, 1978). Ces « sens partiels » ou «membres de la phrase » peuvent facilement recouvrir la notion syntaxique de constituant immédiat (syntagme nominal, syntagme verbal, syntagme prépositionnel, syntagme adverbial, etc.) Ils recouvrent également la notion prosodique de groupe rythmique, ou mot phonétique (Wioland, 2005), ou groupes accentuels. Martin (2013) montre que la structuration prosodique serait la première à intervenir dans le processus de génération de l'énoncé par le locuteur, mais aussi la première à être décodée par l'auditeur. Cette structuration prosodique permettrait ensuite l'accès aux autres structurations de l'énoncé: syntaxiques, morphologiques, informationnelles.

Voici un court exemple de découpage inadéquat rompant la logique du discours. Il s'agit de l'intervention remarquée de Dominique de Villepin au Conseil de sécurité de l'Organisation des Nations Unies à New York, le 14 février 2003. Pour des raisons de clarté (et sans doute de traduction simultanée), l'orateur produit des groupes rythmiques courts, marqués par des pauses et/ou des allongements de dernière syllabe, structurant très clairement son discours. Voici l'extrait qui nous intéresse présenté sans ponctuation.

«Et c'est un vieux pays la France d'un vieux continent comme le mien l'Europe qui vous le dit aujourd'hui. » Nous ne disposons que de la version orale de ce discours (voir fichier son). Le découpage inadéquat réalisé est :

[Et c'est un vieux pays/la France d'un vieux continent comme le mien / l'Europe / qui vous le dit aujourd'hui. //]

34 Le découpage d'un texte écrit en groupes rythmiques, lorsqu'il n'est pas indiqué par la ponctuation, est toujours un point intéressant à discuter en classe. Il peut s'agir de groupes rythmiques minimaux, proches de la notion syntaxique de constituants immédiats, ou de groupes plus longs pour des raisons d'équilibre des groupes à l'intérieur de l'énoncé (la «proportion» de Beauzée, l'hypothèse de l'équilibre temporel de Wioland). Dans tous les cas, le découpage vise bien à «faciliter la compréhension du texte».

La frontière entre deux groupes rythmiques d'un même énoncé n'est pas toujours l'occasion d'une pause silencieuse. L'allongement et/ou l'intonation portée par la 
dernière syllabe du groupe et la rupture rythmique visant à respecter l'équilibre temporel sont autant d'indices de la présence d'une frontière de groupe. Ces paramètres sont à pratiquer pour favoriser l'intelligibilité de la lecture à haute voix.

\section{Identifier les contenus phonétiques d'un texte littéraire}

36 S'il est rare, hormis des poèmes à rimes, de choisir d'emblée un texte littéraire à des fins d'exploitation phonétique, on peut chercher à analyser les caractéristiques phonétiques d'un document écrit. Il s'agit ici tout comme l'analyse linguistique et l'analyse littéraire, d'explorer le texte en détail afin de bien le connaître et d'en sélectionner des contenus récurrents exploitables en classe en fonction du niveau.

\section{1. Analyse suprasegmentale}

37 La première lecture du texte doit permettre d'identifier les groupes rythmiques par des «/» (et «//» en fin d'énoncé). On suivra bien sûr les marques de ponctuation, mais elles sont souvent insuffisantes pour identifier les groupes rythmiques minimaux. Cette première lecture en groupes rythmiques soulèvera quelques questionnements, que l'on retrouvera en classe, dus en particulier à la notion d'équilibre et de proportion entre les groupes. Une façon de résoudre la question est de savoir ce qui permettrait une meilleure compréhension en sachant que le mot à mot n'est pas une option. Cette première étape d'identification des groupes rythmiques nous semble essentielle dans toute première lecture. Elle permet d'éviter l'écueil d'une lecture «en tunnel» en découpant le continuum écrit en unités de sens. Cette activité de segmentation préparatoire à la lecture à haute voix est bénéfique même au locuteur de langue maternelle.

Une fois les groupes rythmiques identifiés, on peut poursuivre l'analyse suprasegmentale en rassemblant les groupes rythmiques par nombre de syllabes : des plus courts aux plus longs. On pourra se servir de ces regroupements pour faire remarquer aux étudiants les similitudes de rythme en dépit du nombre de mots. Par exemple : L'ouverture / de la bouche ${ }^{6}: 3$ syllabes dans les deux cas = un même rythme, 2 mots dans le $1^{\mathrm{er}}, 3$ mots dans le second.

Dans le style oral retranscrit, les groupes rythmiques sont parfois interchangeables comme dans l'exemple suivant: «Il faut qu'elle travaille, ces vacances ${ }^{7}$ ». Lorsqu'elle apparaît, cette caractéristique des groupes rythmiques est à noter.

Les liaisons et les enchaînements consonantiques apparaissant à l'intérieur des groupes rythmiques sont à relever afin d'éventuellement les travailler après les avoir organisés.

41 Pour l'intonation, on veillera à la structure des phrases interrogatives: avec ou sans mot interrogatif. C'est seulement en l'absence de mot interrogatif que l'intonation doit être montante. On relèvera les montées de continuation majeure, les montées de continuation mineure, les parenthèses basses et les finalités afin de hiérarchiser la structure de l'énoncé. On traitera éventuellement à part l'intonation expressive.

Les syllabes accentuées (dernière syllabe de chaque groupe rythmique) sont les syllabes les plus importantes du document. Pour en finir avec l'analyse suprasegmentale, on peut classer les syllabes accentuées en syllabes ouvertes et en syllabes fermées. On ne 
s'étonnera pas de constater un relatif équilibre en nombre entre les deux types de syllabes. En effet, c'est l'analyse de toutes les syllabes en français (inaccentuées et accentuées) qui montre environ $80 \%$ de syllabes ouvertes (dues en particulier aux enchaînements consonantiques) pour $20 \%$ de syllabes fermées. Mais l'analyse des seules syllabes accentuées montre normalement une répartition 50/50 sur de grands corpus (Wioland, 1991).

\section{2. Analyse segmentale}

L'analyse segmentale se fera à partir du relevé des syllabes accentuées en A.P.I. Commençons par les voyelles: on observera la fréquence des voyelles qui posent souvent problème, c'est-à-dire en priorité les voyelles antérieures labiales [y, ø, œ] et

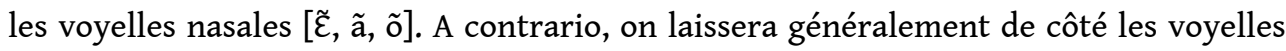
$[\mathrm{i}, \mathrm{e}, \mathrm{a}, \mathrm{o}]$ qui posent rarement problème, sauf dans des cas de forte diphtongaison par exemple. On relativisera l'importance des oppositions des voyelles moyennes $[\mathrm{e} / \varepsilon, \varnothing / œ$, o/ว], sauf si le texte permet d'observer la tendance générale en français : la voyelle la plus fermée en syllabe ouverte, la voyelle la plus ouverte en syllabe fermée.

Pour les consonnes, on observera prioritairement la position forte (à l'initiale de la syllabe accentuée). On relèvera les groupes de consonnes (sans oublier ceux apparus suite à l'effacement d'un <e> à l'oral par exemple). On distinguera pour chaque consonne les distributions en fonction des positions s'il y a lieu. Par exemple, pour [1] ou [R], en position finale absolue, en finale de syllabe avant consonne, en position initiale, intervocalique (entre voyelles), après consonne. Ici aussi, on portera évidemment plus d'attention aux consonnes qui posent problème (plus diverses en fonction des langues d'origine des étudiants).

Une fois une voyelle ou une consonne identifiée comme particulièrement fréquente en syllabe accentuée, on peut en chercher d'autres occurrences en syllabe inaccentuée.

Cette analyse systématique des contenus phonétiques d'un texte littéraire permet à l'enseignant une lecture précise des contenus phonétiques, de préparer les questionnements autour de la structuration syntaxico-prosodique du texte et d'anticiper l'apparition de certaines difficultés de décodage graphie - phonie et de prononciation.

\section{Conclusion}

47 Le texte littéraire est un document authentique présentant de nombreux attraits culturels, stylistiques et linguistiques qui justifie son utilisation en classe de langue à tous les niveaux de l'apprentissage. Parmi les aspects linguistiques, la phonétique est souvent négligée. L'analyse des aspects segmentaux est généralement réservée aux poèmes en rimes. L'analyse des syllabes accentuées d'un texte en prose peut être pourtant tout aussi riche, mettant en valeur les structures syllabiques, les voyelles et les consonnes (simples ou en clusters) susceptibles de poser problème. De même l'analyse prosodique et en particulier le découpage en unités de sens (groupes rythmiques, mots phonétiques, groupes de souffle, etc.) et les questions afférentes (intelligibilité optimale et équilibre rythmique entre les unités), constitue une clé majeure pour accéder au sens général et détaillé d'un texte. C'est aussi une étape préparatoire indispensable à l'oralisation. 


\section{BIBLIOGRAPHIE}

Balzac H. de, 1848, Le cousin Pons. Disponible sur : <https://fr.wikisource.org/wiki/

Le_Cousin_Pons>.

Bernstein S., 1975, « Enseignement de la prononciation russe aux étrangers ", in A. Leontiev et N. Samouïlova (dir.), La phonétique et l'enseignement de la prononciation, Moscou, MGU, p. 5-61.

Blondeau N., Allouache F. et Né M.-F., 2004, Littérature progressive du français (niveau débutant), Paris, CLE International.

Bouchery C. et Taillandier I., 2009, Le français par les textes littéraires, Paris, Belin, coll. « FLE ».

Catach N., 1994, La Ponctuation, Paris, PUF, coll. « Que sais-je?».

Charliac L., Morton A.-C., Le Bougnec J.-T. et Loreil B., 2003, Phonétique progressive du français (niveau débutant), Paris, CLE International.

Chevalier B., 1992, Lecture et prise de notes, Paris, Nathan Université, coll. « 128 ».

Cuq J.-P. et Gruca I., 2002, Cours de didactique du français langue étrangère et seconde, Grenoble, PUG, coll. « Didactique (FLE)».

Defays J.-M., Delbart A.-R., Hammami S. et Saenen F., 2014, La littérature en FLE : état des lieux et nouvelles perspectives, Paris, Hachette FLE, coll. « $\mathrm{f} »$.

Delattre P., 1944, « Vers la méthode phonétique intégrale pour débutants », The French Review, vol. 18, no 2, p. 109-115.

Fiévet M., 2013, Littérature en classe de FLE, Paris, CLE International, coll. « Techniques et pratiques de classe ».

Filliolet C. et Vandendriessche A., 2010, La phonétique par les textes, Paris, Belin, coll. « FLE ».

Gadet F., 1989, Le Français ordinaire, Paris, Armand Colin.

Grévisse M., 1993, Le bon usage, 13e édition, Paris, Duculot.

Lah M., 2014, « La lecture à haute voix en classe de langue étrangère - une activité à réhabiliter? », Linguistica, Ljubljana University Press. Disponible sur : <https://revije.ff.uni-lj.si/ linguistica/article/viewFile/2565/2287>.

Lhote É., 2001, « Pour une didactologie de l'oralité », ÉLA (Études de linguistique appliquée), nos 123-124, p. 445-453. Disponible sur : <https://www.cairn.info/revue-ela-2001-3page-445.htm>.

Lorenceau A., 1978, "Sur la ponctuation au 18e siècle », Dix-huitième Siècle, no 10, dossier «Qu'est-ce que les lumières ?», p. 363-378. Disponible sur : <http://www.persee.fr/doc/ dhs_0070-6760_1978_num_10_1_1195>.

Martin P., 2013, « La structure prosodique opère-t-elle avant ou après la syntaxe ? », TIPA (Travaux interdisciplinaires sur la parole et le langage), no 29, dossier : « Le français parlé » coordonné par Fréderic Sabio. Disponible sur : <http://journals.openedition.org/tipa/ 943>.

Maupassant G. de, 1883, « Un duel ». Disponible sur : <https://fr.wikisource.org/wiki/ Le_Colporteur_(recueil)/Un_duel>. 
Morel M.-A. et Danon-Boileau L., 1998, Grammaire de l'intonation : l'exemple du français, Paris, Ophrys, coll. « Linguistique ».

Pagel D., Wioland F. et Madelini É., 2012, Le rythme du français parlé, Paris, Hachette, coll. « Français Langue Étrangère ».

Peytard J., 1982, « Sémiotique du texte littéraire et didactique du FLE », ÉLA (Études de linguistique appliquée), no 45, p. 91-103.

Puren C., 1988, Histoire des méthodologies de l'enseignement des langues, Paris, Nathan-CLE international, coll. « Didactique des Langues Étrangères ».

Renard R., 1979, La méthode verbo-tonale de correction phonétique, Paris, Didier.

Robert J.-P. (dir.), 2002, Dictionnaire pratique de didactique du FLE, Paris, Orphys.

Weber C., 2013, Pour une didactique de l'oralité. Enseigner le français tel qu'il est parlé, Paris, Didier, coll. « Langues et didactique ».

Wioland F., 1991, Prononcer les mots du français : des sons et des rythmes, Paris, Hachette FLE, coll. «F/autoformation ».

Wioland F., 2005, La vie sociale des sons du français, Paris, L'Harmattan.

\section{NOTES}

1. À propos de la méthodologie traditionnelle.

2. Pour des raisons évidentes de simplicité de passation, les épreuves de base du TCF (Test de Connaissance du Français) sont: compréhension orale, compréhension écrite, maîtrise des structures de la langue.

3. «Lire le texte littéraire, c'est chercher à percevoir les mouvements mêmes du langage là où ils sont les plus forts » (Peytard, $1982: 102$ ).

4. Fiévet, $2013: 41$.

5. Par exemple, les lectures publiques marathons de La Princesse de Clèves, entre 2007 et 2009.

6. Molière, Le Bourgeois Gentilhomme (1670), acte II, scène 4.

7. Françoise Sagan, Bonjour Tristesse (1954), Paris, Julliard.

\section{RÉSUMÉS}

L'exploitation d'un texte littéraire en classe de langue répond le plus souvent à des objectifs de compréhension écrite: accès au sens par la forme/structure du texte et par des éléments linguistiques (lexique, morpho-syntaxe) qu'il faut apprendre à repérer. À cet objectif de compréhension s'ajoutent des objectifs culturels explicites et implicites propres au texte littéraire. La phonétique est la composante de la langue qui semble la moins concernée par l'exploitation du texte littéraire sauf pour la poésie et la lecture à haute voix.

Le présent article, après avoir rappelé les rapports complexes entretenus par l'oral et l'écrit en sciences du langage et en didactique du FLE, décrit les possibles exploitations phonétiques du 
texte littéraire en proposant une procédure d'identification des contenus phonétiques afin de le préparer, au-delà de la simple orthoépie liée à l'écrit, pour faciliter l'accès au sens.

In a language class, the goal of studying a literary text is often written compréhension: understanding meaning through structure, and learning to spot linguistic elements such as vocabulary and syntax. In addition to written comprehension, there may also be explicit and implicit cultural goals specific to a given text. Phonetics is the branch of language that seems the least likely to be concerned with the study of a text, except for poetry and reading aloud.

After having looked at the complex relationship that exists between speech and writing in language study (linguistics) and in teaching French as a Foreign Language, this article describes a way, over and above the study of ordinary pronunciation of a text, to instead identify the phonetic contents of a text that can help make it easier to understand meaning.

\section{INDEX}

Mots-clés : textes littéraires, analyse des contenus phonétiques.

Keywords : literary texts, analysis of the phonetic contents.

\section{AUTEUR}

\section{BERTRAND LAURET}

Institut de Linguistique et de Phonétique Générales et Appliquées Sorbonne Nouvelle-Paris 3 